\title{
Investigation of telomeres in Egyptian patients with bone marrow failure
}

\begin{abstract}
Background and aim for the work: Bone marrow failure (BMF) is the inability of the bone marrow to produce an adequate number of peripheral blood cells. BMF included inherited and acquired conditions. The telomerase complex maintains telomere length (TL) in embryonic cells and in few adult cell types such as germ cells, stem cells, T lymphocytes, and monocytes. All the other adult cells, including blood cells, show a progressive reduction in telomere length, which correlates with the age and rate of cell proliferation. Our study aims to evaluate the utility of Real-Time Quantitative-Polymerase Chain Reaction (RQPCR) in measuring the relative telomere length and to investigate its correlation with the clinicopathological data of the Egyptian BMF patients.
\end{abstract}

Materials and methods: The study included 3 groups: a group of inherited BMF (25 patients), a group of acquired BMF (10 patients) and a third healthy control group (15 cases). The relative telomere length (RTL) is evaluated for them using RQ-PCR.

Results: We have found a significant difference in RTL between all BMF patients and healthy controls $(\mathrm{P}$ value $=0.001)$, there was a significant difference in RTL between inherited BMF patients and its age-matched controls $(\mathrm{P}$ value $=0.009)$, also a significant difference between acquired BMF patients and its age-matched controls ( $\mathrm{P}$ value $=0.034)$. However, there was no significant difference between inherited and acquired BMF patients $(\mathrm{P}$ value $=0.479)$. On the other hand, we find an association between RTL and lymphocyte count $(\mathrm{P}$ value $=0.032)$ in inherited BMF patients. While another association observed with $\mathrm{RTL}$ and red blood cell count $(\mathrm{RBCs})$ in acquired BMF patients $(\mathrm{P}$ value $=0.048)$. There was no significant correlation with RTL and the overall survival or prognosis of those patients.

Conclusion: We conclude that the telomere lengths are significantly altered in Egyptian BMF patients, either whether inherited or acquired compared to the control group, with some hematological changes.

Keywords: telomere length, telomerase, telomere shortening, bone marrow failure (BMF), real-time quantitative PCR (RQ-PCR)
Volume 7 Issue 3 - 2019

\author{
Zainab H El-Dahshan,' Mohammed M \\ El-Naggar,' Om AliY. El-Khawaga,' Ahmed \\ El-Waseef,' 'Sherin Abd El-Aziz, ${ }^{2}$ Hosam \\ Zaghloul, ${ }^{2}$ Ahmad Darwish, ${ }^{3}$ Hasan Abdel- \\ Ghaffar, ${ }^{2}$ Mohamed Mabed ${ }^{4}$ \\ 'Biochemistry Division, Chemistry Department, Faculty of \\ Science, Mansoura University, Egypt \\ ${ }^{2}$ Department of Clinical Pathology, Faculty of Medicine, \\ Mansoura University, Egypt \\ ${ }^{3}$ Department of Pediatric, Children's Hospital, Faculty of \\ Medicine, Mansoura University, Egypt \\ ${ }^{4}$ Hematology Unit, Oncology Center, Faculty of Medicine, \\ Mansoura University, Egypt
}

Correspondence: Mohamed Mabed, Oncology Center, Faculty of Medicine, Mansoura University, Egypt, Postal code: 35516, Emailmohmbed@mans.edu.eg

Received: August 20,2019 | Published: November 06, 2019

\section{Introduction}

Bone marrow failure (BMF) included clinically or pathologically causes associated with the inability of the bone marrow to produce a sufficient number of peripheral blood cells, and it is also called aplastic anemia (AA). BMF includes both inherited and acquired conditions. Inherited bone marrow failure syndromes (IBMFS), such as Dyskeratosis Congenita (DC), Fanconi anemia (FA), DiamondBlackfan anemia (DBA), and Shawchman Diamond syndrome (SDS), are more common to children, but on contrary acquired AAs are more frequent in adults. ${ }^{1}$ The telomerase complex maintains telomere length (TL) in embryonic cells and in few adult cell types such as germ cells, stem cells, T lymphocytes, and monocytes. All the other adult cells, including blood cells, show a progressive reduction in telomere length, which correlates with the age and rate of cell proliferation. ${ }^{2}$ The telomerase complex includes the catalytic subunit (TERT), its RNA component TERC, and the four H/ACA RNA associated proteins dyskerin, NOLA1 (GAR1), NOLA2 (NHP2), and NOLA3 (NOP10). ${ }^{3}$ Without telomerase, telomeres get shorter as the cell duplicates and their erosion induces chromosomal instability and apoptosis.
Telomere DNA consists of long repeated sequences of (TTAGGG) on the ends of chromosomes which needed for the replication and stability of chromosomes. Telomeres generally shortened with age in somatic cells and, in well-known, telomere shortening encountered by telomerase activity to keep up TL, and this commonly found between specific cells, particularly in stem cells. ${ }^{4}$ There are other factors that have an effect on telomere shortening than aging, including oxidative stress, DNA damage, DNA repair mechanisms, and genetic factors. ${ }^{5}$ In normal cells, telomere shortening considered a result of cell senescence or apoptosis. ${ }^{6}$ Critical telomeres shortening that breakout apoptosis or senescence has risky genomes and are believed to be a critical step on the pathway to malignant transformation. ${ }^{7,8}$ Many studies have reported short telomeres in patients with BMF syndromes, using variety of different cell types, and different methods as Southern blot, fluorescence in situ hybridization combined with flow cytometry (flow-FISH), and Quantitative PCR.9-14 So, our study aimed to evaluate the utility of Real-Time Quantitative-Polymerase Chain Reaction (RQ-PCR) in measuring the relative telomere length and investigating its correlation with the clinicopathological data of a cohort of Egyptian patients with BMF syndromes either acquired or inherited. 


\section{Subjects and methods}

\section{Subjects}

This study included 3 groups (50 individuals): 25 patients as inherited BMF group, 10 patients with pure red blood cell aplasia
(PRBCA), 15 patients with Fanconi anemia (FA), while 10 patients in acquired BMF group as acquired aplastic anemia (AAA) and 15 normal individuals as a third group 10 as age-matched healthy individuals for inherited BMF group (ranges from 3 to 17 years old) and 5 as age-matched individuals for acquired BMF group (ranges from 21-70 years old). Patient characteristics are shown in Table 1.

Table I Demographic, laboratory data and Patient characteristics.

\begin{tabular}{|c|c|c|c|c|}
\hline & & Control group & $\begin{array}{l}\text { Inherited BMF } \\
\text { patients }\end{array}$ & $\begin{array}{l}\text { Acquired BMF } \\
\text { patients }\end{array}$ \\
\hline $\mathrm{N}$ & & 15 & 29 & 10 \\
\hline SEX & $M / F$ & 09-Jun & $16 / 13$ & 05-May \\
\hline AGE & Median (Range) & $35(3-70)$ & $6(1-16)$ & $34(21-70)$ \\
\hline $\mathrm{HB}(\mathrm{g} / \mathrm{dL})$ & Median (Range) & I2.I (I0.0-14.30) & $7.29(3.92-11.10)$ & $7.75(4.18-9.41)$ \\
\hline $\operatorname{RBCs}(x|0| 2 / L)$ & Median (Range) & $5.0(4.47-5.54)$ & $2.54(1.49-5.12)$ & $2.83(1.90-4.18)$ \\
\hline WBCs(xI09/L) & Median (Range) & $6.50(5.50-7.10)$ & $5.56(0.99-32.80)$ & $3.56(1.14-4.83)$ \\
\hline LYM\% & Median (Range) & $63.0(34.20-76.50)$ & 44.5 (I5.20-75.20) & $63.0(29.60-76.50)$ \\
\hline NEUT\% & Median (Range) & $50.0(40.0-55.0)$ & $35.90(10.0-79.60)$ & $27.10(18.6-69.0)$ \\
\hline PLTs (x 109/L) & Median (Range) & $195.0(190.0-313.0)$ & $166.0(4.30-\mid$ I 79.0$)$ & 28.1 (II.I0-37I.0) \\
\hline
\end{tabular}

BMF, bone marrow failure; HB, hemoglobin; RBCs, red blood cells;WBCs. white blood cells; PLTs, platelets; LYM, lymphocytes

BMF patients were diagnosed based on complete blood count and bone marrow aspiration and biopsy examination. Patients with inherited BMF syndromes included pure red blood cell aplasia (PRBCA) and Fanconi anemia (FA), these two syndromes were diagnosed according to clinical criteria, including specific congenital anomalies and syndrome-specific laboratory tests; ${ }^{15}$ chromosome breakage analysis for FA, and red cell adenosine (ADA) for PRBCA. Acquired BMF patients were diagnosed according to the presence of the bone marrow hypo cellular without congenital anomalies and negative results of chromosomal breakage analysis.

Inherited BMF patients followed a protocol of treatment for androgens and corticosteroids; most of them were blood transfusion dependent. Four patients that received a successful bone marrow transplant (BMT) showed slightly improved levels of hematology parameters. Patients with acquired BMF were treated with cyclosporin A (CsA). Also, most of them were blood transfusion dependent. Patients were recruited from the hematology outpatient clinic at Children Hospital (MUCH) for children and for adults at Oncology Centre Mansoura University (OCMU), Mansoura, Egypt. Written informed consent was obtained from all participants. This study was approved by the Human Ethics Committee of the Mansoura University in accordance with the Declaration of Helsinki.

\section{Measurement of Telomere by RQ-PCR}

Genomic DNA extracted from $200 \mu \mathrm{L}$ of peripheral blood using Gene JETTM Genomic DNA Purification Kit (Fermentas spin columns, Canada).Extracted DNA carried on different occasions. The relative average of telomere length (RTL) determined by RQ-PCR as described by Cawthon ${ }^{16}$ with some changes. For each DNA sample, telomere (T) and single copy gene (S) master mixes prepared and ran on Applied Biosystems StepOne ${ }^{\mathrm{TM}}$ (Applied Biosystems, Foster City, CA, USA). The reaction mixture was in a final volume of 30 $\mu \mathrm{L}$ containing $12 \mu \mathrm{L}$ SYBR Green master mix (Applied Biosystems, Warrington, UK, 4344464) and 35ng of DNA, these also included the final concentrations for telomere primer $270 \mathrm{nM}$ for tel1; and $900 \mathrm{nM}$ for tel2, and $300 \mathrm{nM}$ for $36 \mathrm{~B} 4 \mathrm{u}$; and $500 \mathrm{nM}$ for $36 \mathrm{~B} 4 \mathrm{~d}$ (single copy gene). The 36B4 gene encodes acidic ribosomal phosphoprotein PO, is on chromosome $12,{ }^{17}$ (written, (Table 2 ) were:

Table 2 Primer sequences for quantitative real-time PCR.

\begin{tabular}{lll}
\hline Name & $\begin{array}{l}\text { Primer sequences } \\
\mathbf{( 5 \rightarrow 3 )}\end{array}$ \\
\hline Telomere & tell, GGTTTTTGAGGGTGAGGGTGAGGGTGAGGGTGAGGGT \\
& Reverse & tel2,TCCCGACTATCCCTATCCCTATCCCTATCCCTATCCCTA \\
$36 \mathrm{~B} 4$ & Forward & \\
& Reverse & 36B4d, CAGCAAGTGGGAAGGTGTAATCC \\
\hline
\end{tabular}


Each run involved two 96-well plates prepared for each assay, one containing telomere primers and the other 36B4 primers used as a single copy gene. A standard DNA (Applied Biosystems, Foster City, CA, 350436) was serially diluted by $\sim 1.68$-fold per dilution to produce five concentrations of DNA, ranging from $0.63 \mathrm{ng}$ to $5 \mathrm{ng} / \mu \mathrm{L}$ to produce standard curves one for telomere repeats and the other for 36B4 gene. Then, efficiency calculated by using the formula $\mathrm{E}=10$ [-1/slope] -1 . The slope values for the telomere and 36B4 assays in the current study were -3.289 and -3.314 respectively; generating efficiencies equals 99\% (recommended range 90-110\%). All samples analyzed in triplicates. Amplification was carried out at $95^{\circ} \mathrm{C}$ for 10 min followed by for telomere primer, 18 cycles at $95^{\circ} \mathrm{C}$ for $15 \mathrm{~s}, 54^{\circ} \mathrm{C}$ for $2 \mathrm{~min}$. For 36B4 primer followed by 30 cycles at $95^{\circ} \mathrm{C}$ for $15 \mathrm{~s}$, $58^{\circ} \mathrm{C}$ for $1 \mathrm{~min}$. Telomere/Single-copy gene (T/S) ratio values were then calculated using the formula for comparative method $\mathrm{T} / \mathrm{S}=2$ $\Delta \Delta \mathrm{CT}$ for relative quantification. $\mathrm{T} / \mathrm{S}$ ratios for each sample obtained by dividing the mean amount of telomeres by the mean amount of 36B4.

Statistical Analysis: Statistical analysis performed with SPSS version 16.0. Non-parametric tests were used. Median and Range calculated for telomere length. Mann-Whitney Rank Sum test is a non-parametric test used to compare two populations' means that come from the same population. The Kruskal-Wallis $\mathrm{H}$ test used to compare between two or more groups. The Kendall rank correlation coefficient used to assess the association of $\mathrm{T} / \mathrm{S}$ ratio measurements with the study variables. Receiver`s Operating Characteristics (ROC) curve analysis used to get the area under the curve (AUC) to decide the diagnostic power of RTL in BMF patients either inherited or acquired. Statistical significance was considered when $\mathrm{P}<0.05$.

\section{Results}

A total 35 patients with BMF and 15 individuals as healthy age-matched control group were included in this study. RTL was significantly lower in all patients with BMF (Median: -2.30, range: -5.33 to -0.09 ) than that of all age-matched controls (Median: -1.37 , range: -1.74 to -1.01$),(\mathrm{P}<0.001)$. Meanwhile, there was a significant difference between inherited BMF patients (Median: -2.30, range: -4.49 to -0.09 ) and its age-matched controls (Median: -1.53 , range: -1.74 to -1.01$),(\mathrm{P}<0.009)$. Also, there was a significant difference between acquired BMF patients (Median: -1.82 , range: -5.33 to $-0.49)$ and their controls (Median: -1.30 , range: -1.60 to -1.01$),(\mathrm{P}<$ $0.034)$. On the other hand, there was no significant difference in both inherited (Median: -2.30 , range: -4.49 to -0.09 ) and acquired BMF patients (Median: -1.82 , range: -5.33 to -0.49$)$, $(\mathrm{P}>0.479)$. There was no significant difference in RTLs between different subtypes of inherited BMF patients $(n=29, P>0.267)$ (Table 3$)$.

From the ROC curve analysis, the supposed optimal cut-off value is 0.032 (for discriminating RTL in all BMF patients from all controls), which yielded a sensitivity of 0.769 , a specificity of 0.600 (1-specificity $=0.400)$ and the AUC is 0.790 (95\% CI: 0.667 to 0.912 ) (Figure 1). While, the supposed optimal cut-off value is 0.014 (for discriminating RTL in the inherited BMF patients from the acquired), which yielded a sensitivity of 0.600 , a specificity of 0.759 (1-specificity $=0.241)$, and the AUC is 0.576 (95\% CI: 0.359 to 793$)$ (Figure 2).

TL has a significant correlation with red blood cells count in acquired BMF patients $(\mathrm{P}<0.048$, Figure 3$)$; however, it has a significant correlation with the lymphocytes in the inherited BMF patients $(\mathrm{P}<0.032$, Figure 4$)$. We observed that there was no association between RTL and patient's situation at last contact neither in acquired BMF patients $(\mathrm{r}=0.031, \mathrm{P}>0.815)$, nor in inherited BMF patients $(\mathrm{r}=0.091, \mathrm{P}>0.583$ ). Also, there was no association between RTL and overall survival from diagnosis neither in acquired BMF patients $(\mathrm{r}=0.090, \mathrm{P}>0.719)$ nor in inherited BMF patients $(\mathrm{r}=0.151$, $\mathrm{P}>0.287$ ) (Table 4).

Table 3 Telomere length comparison in different study groups

\begin{tabular}{|c|c|c|c|}
\hline & Log RTL median & Range & P-value \\
\hline \multirow[t]{2}{*}{ All BMF patients $(n=39)$ vs. Healthy controls $(n=15)$} & $-2.30 v s .-1.37$ & $-5.33:-0.09$ & $0.001 * * *$ \\
\hline & & -I.74:- - .0I & \\
\hline \multirow[t]{2}{*}{ Inherited BMF patients $(n=29)$ vs. age-matched controls $(n=10)$} & $-2.30 v s .-1.53$ & $-4.49:-0.09$ & $0.009 * *$ \\
\hline & & $-1.74:-1.01$ & \\
\hline \multirow[t]{2}{*}{ Acquired BMF patients $(n=10)$ vs. age-matched controls $(n=5)$} & $-1.82 v s .-1.30$ & $-5.33:-0.49$ & $0.034 *$ \\
\hline & & $-1.60:-1.01$ & \\
\hline \multirow[t]{2}{*}{ Inherited $(n=29)$ vs. Acquired $(n=10)$ BMF patients } & $-2.30 v s .-1.82$ & $-4.49:-0.09$ & 0.479 \\
\hline & & $-5.33:-0.49$ & \\
\hline
\end{tabular}

BMF, bone marrow failure; Log RTL: logarithm transformation of $2^{-\Delta \Delta}$ of relative telomere length. ${ }^{18}$ Mann-Whitney $U$ test was used. The significance level was $*$ $p \leq 0.05$, ** $p \leq 0.0$ I or $* * * p \leq 0.00$ I. 


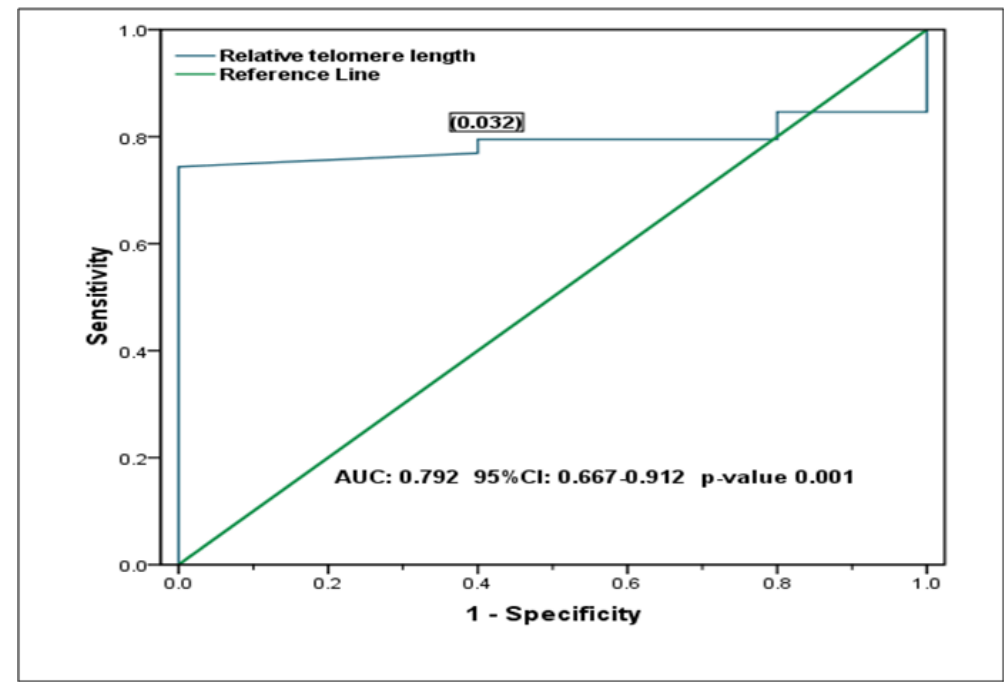

Figure I Receiver operating characteristic (ROC) curve analysis shows the relative telomere length (RTL) in all BMF patients with all healthy controls: The supposed optimal cutoff value of RTL is 0.032 used as a marker for discriminating BMF patients from healthy controls.

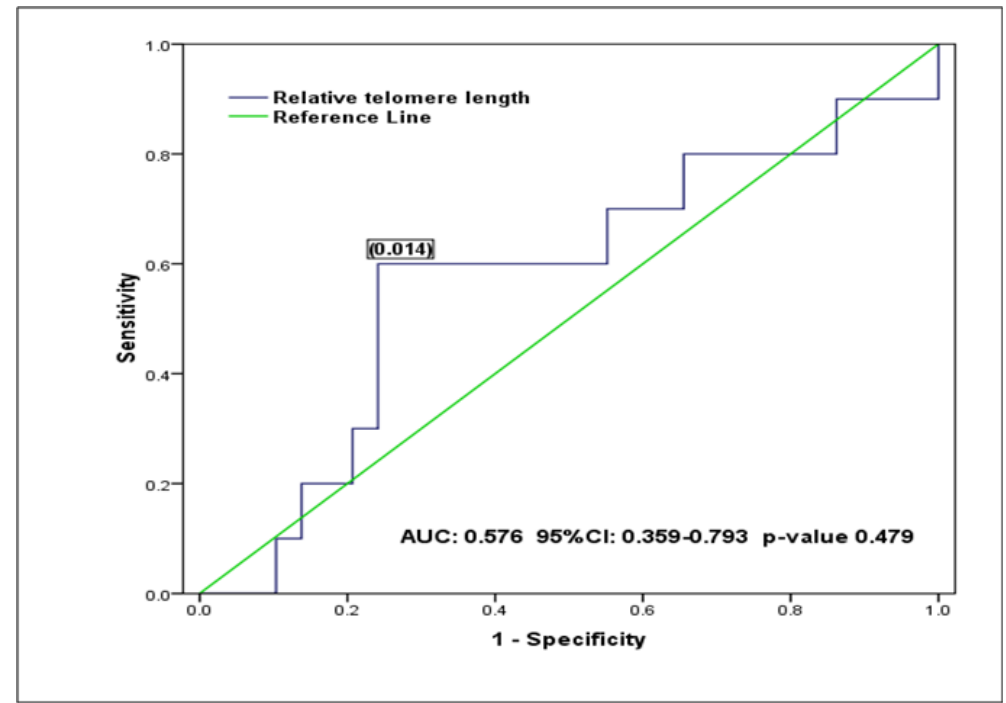

Figure 2 Receiver operating characteristic (ROC) curve analysis shows the relative telomere length (RTL) in inherited and acquired BMF patents: The supposed optimal cutoff value of RTL is 0.014 used as a marker for discriminating inherited BMF group from the acquired.

Table 4 Correlations of Relative Telomere Length (RTL) in BMF patients with their clinicopathological status.

\begin{tabular}{llllll}
\hline Items & \multicolumn{2}{l}{$\begin{array}{l}\text { Acquired BMF patients } \\
\mathbf{n = 1 0}\end{array}$} & $\begin{array}{l}\text { Inherited BMF patients } \\
\mathbf{n = 2 9}\end{array}$ \\
\hline & & R-value & p-value & R-value & p-value \\
\cline { 2 - 6 } & Age & -0.045 & 0.857 & -0.167 & 0.218 \\
& Sex & -0.06 & 0.834 & -0.021 & 0.895 \\
& HB & 0 & 1 & 0.08 & 0.553 \\
& RBCs & 0.494 & $0.048^{*}$ & 0.106 & 0.429 \\
RTL & -0.18 & 0.472 & -0.013 & 0.921 \\
& PLT & -0.25 & 0.321 & 0.077 & 0.567 \\
& WBCs: & -0.225 & 0.369 & -0.339 & $0.032^{*}$ \\
& -Lymphocytes & 0.135 & 0.59 & -0.07 & 0.674 \\
& -Monocytes & 0.09 & 0.719 & 0.076 & 0.649 \\
& -Neutrophiles & 0.366 & 0.173 & -0.1 & 0.537 \\
& -Eosinophils & -0.366 & 0.173 & 0.15 & 0.384 \\
& -Basophils & 0.031 & 0.815 & 0.091 & 0.583 \\
& -PCS & 0.09 & 0.719 & 0.151 & 0.287 \\
\hline
\end{tabular}

BMF, bone marrow failure; $\mathrm{HB}$, hemoglobin; RBCs, red blood cells;WBCs, white blood cells; $\mathrm{PCS}$, patient's current situations and OAS: overall survival.The Kendall rank correlation coefficient was used where appropriate. The significance level was $* p \leq 0.05$, *** $p \leq 0.01$ or $* * * p \leq 0.00$ I. 


\section{Discussion}

It is worth mentioning that we are in Egypt in the Middle East area, there is a difference in lengths of telomeres. To evaluate the differences in the TL on multi-ethnic groups, non-Hispanic Whites were found to have shorter TL compared to Caucasians,${ }^{19}$ African Americans,${ }^{20}$ and Hispanics. ${ }^{5,21}$ Whilst, another study suggested that African Americans and Hispanics have a shorter TL than Non-Hispanic Whites. ${ }^{22}$ Other factors confirmed a relationship between telomere repeats in certain multi-ethnic groups as socioeconomic and psychosocial factors which led to variation in telomere shortening, ${ }^{22}$ namely education degree ${ }^{23}$ and perceived strain. ${ }^{24}$

Racism can contribute to being a part of racial variations in $\mathrm{TL},{ }^{25}$ where, the TL is longer in blacks than whites, telomere shortening, is also significantly greater in blacks where they become more aging than whites suggesting health disparities..$^{25}$ Heritability of TL has been said as an opportunity attention in racial variations. ${ }^{7}$ A third study proposed the weathering hypothesis with exposure to chronic stress make contributions to expand telomere shortening, which also causes racial variations. ${ }^{27}$

In our study, TLs in BMF patients were compared to the TLs in the healthy controls observing that BMF patients have shorter telomeres (Table 3). ROC curve analysis showed the diagnostic value of RTL and succeeded to discriminate between BMF patients and healthy controls (Figure 1), but it failed to discriminate RTL between the inherited and acquired BMF patients showing similarities in TL within different subtypes of disease (Figure 2). Our findings are similar to Ball and colleagues and Pavesi and colleagues ${ }^{9,12}$ who reported that BMF patients have shorter telomeres than that of controls and there is no significant difference in TL either inherited or acquired BMF conditions.

The role of TL in BMF patients and its correlation with the clinicopathological data have not yet been delineated. To the best of our knowledge, this is the first time to observe that there is a positive correlation between relative telomere length and red blood cells count reduction in acquired BMF patients (Table 4) (Figure 3). This could be due to that most patients are blood transfusion-dependent, where short telomeres in nucleated RBC progenitors leading to promote telomerase activity by erythropoietin to increase RBCs production. ${ }^{29}$ On the other hand, the findings observed by Ball and colleagues; ${ }^{9}$ which applied to acquired aplastic anemia patients using the Southern blot method, they found that there was no correlation between telomere shortening and recovery of blood elements.

On the other hand, an inverse correlation observed between the lymphocytes count and telomere shortening in inherited BMF patients (Table 4) (Figure 4). This could be explained by the fact that the purified candidate stem cells show asymmetric cell divisions in vivo and variations in the replicative history between individual stem cells seem to show the proliferation capacity on the level of multipotent hematopoietic stem cells where telomerase enzyme is present to encounter telomere shortening. ${ }^{30}$ Meanwhile, a study by Brümmendorf and colleagues ${ }^{31}$ found that there was no correlation between telomere shortening and lymphocytes count in patients with aplastic anemia using the flow-FISH method. Moreover, Alter and colleagues ${ }^{32}$ showed that telomere shortening in lymphocytes associated with severity of BMF in patients with DC, while our study does not include DC

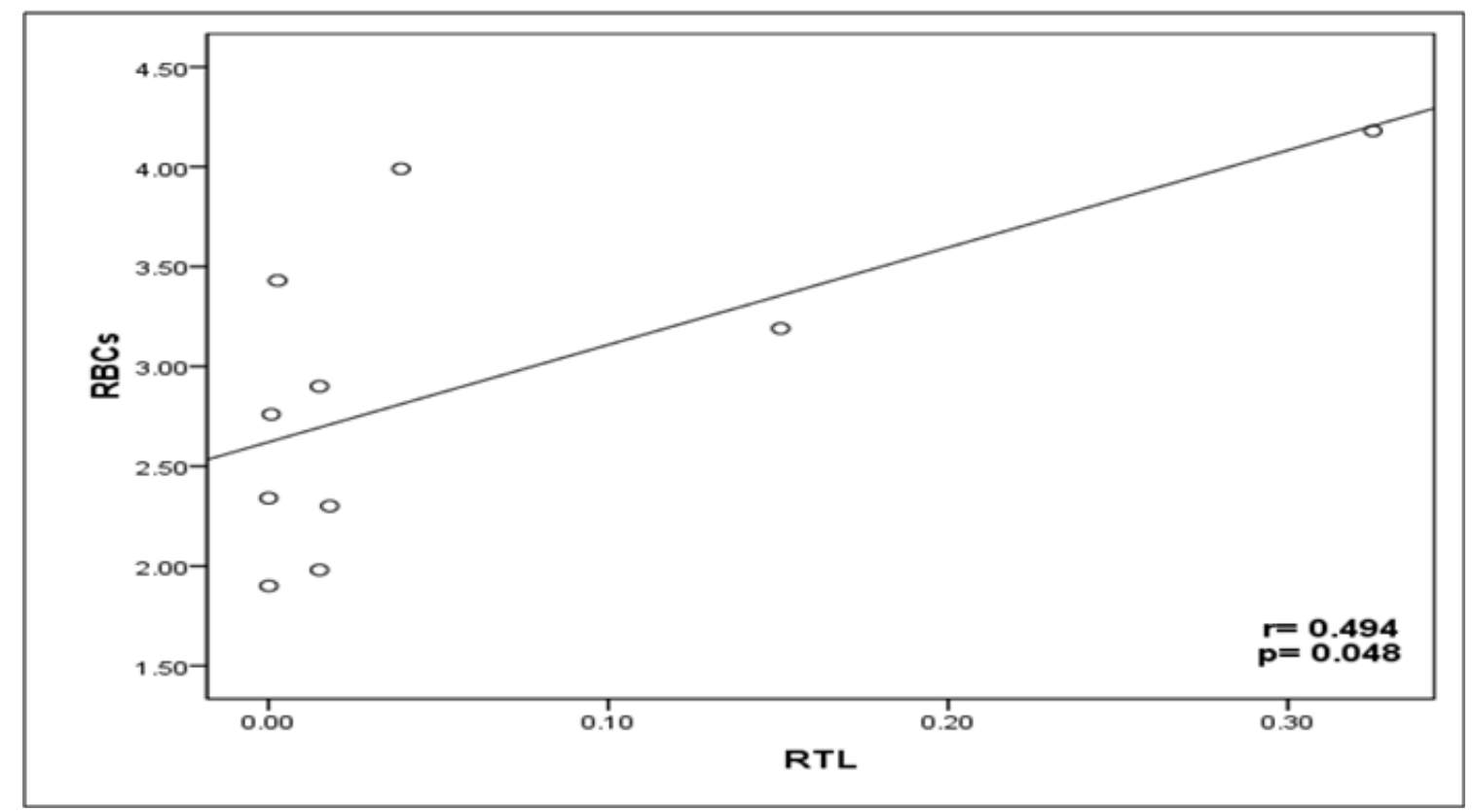

Figure 3 The Kendall's rank correlation coefficient shows the correlation between the Relative Telomere Length (RTL) and Red Blood Cells (RBCs) Count in Acquired Bone Marrow Failure (ABMF) patients: A scatter Plot Shows positive correlation between RTL and RBCs Count $(r=0.494, P<0.05)$. 


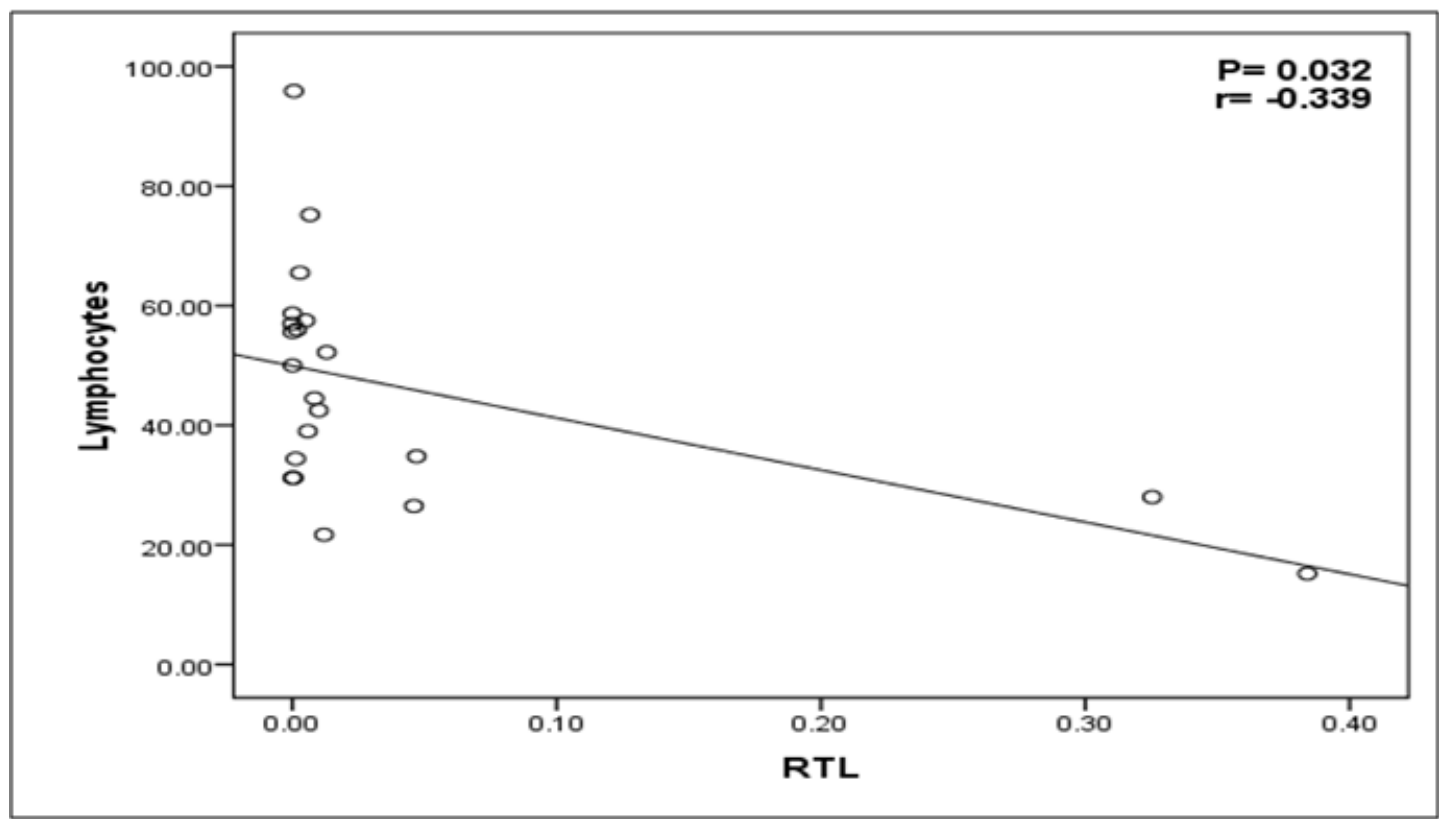

Figure 4 The Kendall's rank correlation coefficient shows the correlation between the Relative Telomere Length (RTL) and Lymphocytes Count in Inherited Bone Marrow Failure patients (IBMF):A scatter plot shows a negative correlation between RTL and Lymphocytes $(r=-0.339, P<0.05)$.

\section{Conclusion}

Telomere length is significantly altered in Egyptian patients with BMF syndromes, whether being inherited or acquired compared to their age-matched controls. We found an inverse correlation between telomere shortening and lymphocytes count in inherited BMF patients, while the telomere length has a positive correlation with red blood cells count in acquired BMF patients. Meanwhile, the degree of telomere shortening does not have relation to patient's prognosis or overall survival.

\section{Acknowledgments}

None.

\section{Conflicts of interest}

The author declares no conflicts of interest.

\section{Funding}

None.

\section{References}

1. Savage SA, Alter BP. The role of telomere biology in bone marrow failure and other disorders. Mechanisms of ageing and development. 2008;129(1):35-47.

2. Lansdorp PM. Telomeres, stem cells, and hematology. Blood. 2008;111(4):1759-1766.

3. Calado RT, Young NS. Telomere diseases. New England Journal of Medicine. 2009;361(24): 2353-2365.

4. Cheung A, Deng W. Telomere dysfunction, genome instability and cancer. Front Biosci. 2008;13(1): 2075-2090. 2741
5. Sanders JL, Newman AB. Telomere length in epidemiology: a biomarker of aging, age-related disease, both, or neither? Epidemiologic reviews. 2013;35(1):112-131.

6. Palm W, de Lange T. How shelterin protects mammalian telomeres. Annual review of genetics. 2008;42: 301-334.

7. Koorstra JB, Hustinx SR, Offerhaus GJ, et al. Pancreatic Carcinogenesis. Pancreatology. 2008;8(2):110-125.

8. Cunningham JM, 22Johnson\%20RA"Johnson RA, 22Litzelman\%20 K"Litzelman K, et al. Telomere length varies by DNA extraction method: implications for epidemiologic research. Cancer Epidemiol Biomarkers Prev. 2013;22(11):2047-2054.

9. Ball SE, 22Gibson\%20FM"Gibson FM, 22Rizzo\%20S"Rizzo S, et al. Progressive telomere shortening in aplastic anemia. Blood. 1998;91(10):3582-3592.

10. Leteurtre F, Li X, Guardiola P, et al. Accelerated telomere shortening and telomerase activation in Fanconi's anaemia. British journal of haematology. 1999;105(4):883-893.

11. Thornley I, Dror Y, Sung L, et al. Abnormal telomere shortening in leucocytes of children with Shwachman-Diamond syndrome. $\mathrm{Br} J$ Haematol. 2002;117(1):189-192.

12. Pavesi E, Avondo F, Aspesi A, et al. Analysis of telomeres in peripheral blood cells from patients with bone marrow failure. Pediatric blood and cancer. 2009;53(3):411-416.

13. Du HY, Pumbo E, Ivanovich J, et al. TERC and TERT gene mutations in patients with bone marrow failure and the significance of telomere length measurements. Blood. 2009;113(2):309-316.

14. Myers KC, Bolyard AA, Otto B, et al. Variable clinical presentation of Shwachman-Diamond syndrome: update from the North American Shwachman-Diamond syndrome registry. J Pediatr. 2014;164(4):866870. 
15. Camitta BM, Thomas ED, Nathan DG, et al. Severe aplastic anemia: a prospective study of the effect of early marrow transplantation on acute mortality. Blood. 1976;48(1):63-70.

16. Cawthon RM. Telomere measurement by quantitative PCR. Nucleic acids research. 2002;30(10):e47.

17. Boulay J, Reuter J, Ritschard R, et al. Gene dosage by quantitative realtime PCR. Biotechniques. 1999;27(2):228.

18. Kubista M, Andrade JM, Bengtsson M, et al. The real-time polymerase chain reaction. Mol Aspects Med. 2006;27(2):95-125.

19. Zhu H, Wang X, Gutin B, et al. Leukocyte telomere length in healthy Caucasian and African-American adolescents: relationships with race, sex, adiposity, adipokines, and physical activity. $J$ Pediatr. 2011;158(2):215-220.

20. Shalev I, Entringer S, Wadhwa PD, et al. Stress and telomere biology: a lifespan perspective. Psychoneuroendocrinology. 2013;38(9):18351842 .

21. Lynch SM, Peek MK, Mitra N, et al. Race, ethnicity, psychosocial factors, and telomere length in a multicenter setting. PloS one. 2016;11(1):e0146723

22. Diez Roux AV, Ranjit N, Jenny NS, et al. Race/ethnicity and telomere length in the Multi-Ethnic Study of Atherosclerosis. Aging cell. 2009;8(3):251-257.

23. Starkweather AR, Alhaeeri AA, Montpetit A, et al. An integrative review of factors associated with telomere length and implications for biobehavioral research. Nurs Res. 2014;63(1):36.

24. Parks CG, Miller DB, McCanlies EC, et al. Telomere length, current perceived stress, and urinary stress hormones in women. Cancer Epidemiol Biomarkers Prev. 2009;18(2):551-560.

25. Chae DH, Nuru-Jeter AM, Adler NE, et al. Discrimination, racial bias, and telomere length in African-American men. Am J Prev Med. 2014;6(2):103-111.

26. Rewak M, Buka S, Prescott J, et al. Race-related health disparities and biological aging: does rate of telomere shortening differ across blacks and whites? Biol Psychol. 2014;99:92-99.

27. Geronimus AT, Hicken M, Keene D, et al. Weathering and age patterns of allostatic load scores among blacks and whites in the United States. Am J Public Health. 2006;96(5):826-833.

28. Aubert G, Hills M, Lansdorp PM. Telomere length measurementCaveats and a critical assessment of the available technologies and tools. Mutation Research/Fundamental and Molecular Mechanisms of Mutagenesis. 2012;730(1):59-67.

29. De Meyer T, De Buyzere ML, Langlois M, et al. Lower red blood cell counts in middle-aged subjects with shorter peripheral blood leukocyte telomere length. Aging cell. 2008;7(5):700-705.

30.Zhou J, Shen X, Huang J, et al. Telomere length of transferred lymphocytes correlates with in vivo persistence and tumor regression in melanoma patients receiving cell transfer therapy. J Immunol. 2005;175(10):7046-7052.

31. Brümmendorf TH, Maciejewski JP, Mak J, et al. Telomere length in leukocyte subpopulations of patients with aplastic anemia. Blood. 2001;97(4):895-900

32. Alter BP, Giri N, Savage SA, et al. Telomere length in inherited bone marrow failure syndromes. Haematologica. 2015;100(1):9-54. 